\title{
Productos y servicios informacionales como evidencia de la aplicación de procesos de gestión de la información y del conocimiento en centros de información
}

\author{
Zunilka Limonta Favier \\ Evelyn Milagro Otero Caballero \\ Elizabeth Álvarez Cambas \\ Centro de Información y Gestión Tecnológica - CIGET Guantánamo, Cuba
}

Yaima Ripoll Moreno

Centro de Información y Gestión Tecnológica - CIGET Granma, Cuba

CASE REPORT

\begin{abstract}
Resumen
Se analiza la gestión de la información y del conocimiento como factores determinantes en el logro de los objetivos organizacionales del Centro de Información y Gestión Tecnológica (CIGET) de Guantánamo, Cuba. Para el caso se abordaron ambos procesos como enfoques unificados e interdependientes. Los efectos de la aplicación de estos enfoques se traducen y se evidencian en cuatro productos y servicios informacionales generados por el CIGET.

Palabras clave

Gestión de la información ; Gestión del conocimiento ; Productos informacionales ; Servicios informacionales ; Estudios de caso ; Centro de Información y Gestión Tecnológica ; Guantánamo ; Cuba
\end{abstract}

\section{Informational products and services as evidence of the implementation of information and knowledge management processes in information centers}

\begin{abstract}
Analyzes the management of information and knowledge as determinants in achieving organizational goals of the Center for Information and Technology Management (CIGET) Guantanamo, Cuba factors. In the case both processes as unified and interdependent approaches were discussed. The effects of the application of these approaches are translated and are evident in four products and informational services generated by the CIGET.
\end{abstract}

Keywords

Information management ; Knowledge management ; Informational products ; Informational services ; Case studies ; Centro de Información y Gestión Tecnológica ; Guantánamo ; Cuba

\section{Introducción}

El crecimiento de la población humana obliga a la redefinición de estrategias para la supervivencia de los seres vivos, para lo cual el hombre utiliza las tecnologías y las ciencias, explota sus ideas, y pone en práctica su sentido común para adaptarse a la realidad cambiante del medio ambiente, todo en aras de mejorar su calidad de vida, usando como base la información.

Actualmente las organizaciones, con o sin acceso a las ventajas que proporcionan las nuevas tecnologías de información y comunicación, realizan diferentes procesos con el fin de cumplir sus objetivos de trabajo, para posicionarse en el mercado y superar a su competencia, algunos de ellos son: la gestión del conocimiento, la 
gestión de información, la vigilancia tecnológica, la inteligencia empresarial entre otros. Estos procesos permiten además a las organizaciones diseñar diversos productos y servicios que se ponen a disposición de sus usuarios y/o clientes para satisfacer sus necesidades.

Cuba viene apostando en la presente década por el fomento de la gestión de la información y la gestión del conocimiento, lo cual se evidencia en importantes iniciativas sociales como: la Política Económica y Social del Partido Comunista Cubano, la Política Nacional de Información, y el Proceso de Perfeccionamiento Empresarial.

A continuación se aborda el caso del Centro de Información y Gestión Tecnológica (CIGET) de Guantánamo (Cuba). Definimos en un primer momento algunas generalidades de la gestión de la información y de la gestión del conocimiento. Seguidamente se presentan las expresiones identificadas de estos procesos, y finalmente se comentan cuatro productos y servicios elaborados en el CIGET Guantánamo en esta perspectiva.

\section{Generalidades de la gestión de la información y de la gestión del conocimiento}

Dos son los conceptos o enfoques centrales de nuestro análisis: la gestión de la información y la gestión del conocimiento.

\subsection{Gestión de la información (Gl)}

Se considera que la gestión de la información es toda acción que se realice donde medie la información, que tenga como salida un "bien" de cualquier tipo, consolidado en una fuente determinada, en tanto se le dé respuesta a la necesidad del usuario (interno o externo) y se culmine con la satisfacción del mismo.

Con la GI pueden entregarse productos en formato papel o digital, ya que ésta permite la obtención de información de interés que soluciona problemas y que sienta las bases para el correcto funcionamiento de la organización, si se le da un uso adecuado.

La GI vista en conjunto es el resultado de la suma del especialista, con la fuente, con un formato, con el tiempo y por supuesto la información, donde se tiene como premisa la resolución de la necesidad del usuario/cliente y la obtención de su satisfacción.

\subsection{Gestión del conocimiento (GC)}

La GC se dedica a la gestión, análisis, búsqueda, distribución, localización y extracción de información relacionada con la empresa, ya sea económica, de negocios, etc. para lograr el éxito organizacional. Lo más importante de ella es que el conocimiento radica en las personas y de ahí se puede extraer y sociabilizar.

La GC es analizada por cada organización según los procesos que en ella se desarrollen, los factores que la condicionan (políticos, sociales, económicos, etc.) y acorde a su propósito social.

Según Piloto Farrucha (2009, p.31) el proceso de Gestión del Conocimiento considera tres elementos importantes: producción, validación e integración del conocimiento: donde la producción tiene que ver con el proceso de creación del nuevo conocimiento, la validación se refiere a la revisión o certificación del conocimiento producido previamente a su integración a la organización; y la integración está dada por el conjunto de actividades orientadas a insertar el nuevo conocimiento en los diferentes procesos organizacionales, con lo que se enriquece y amplía el conocimiento individual y organizacional.

\section{Metodología}

Se realizó un análisis del trabajo realizado en el Centro de Información y Gestión Tecnológica (CIGET) Guantánamo desde su creación hasta finales del 2013, en cuanto a los productos y servicios de información que se prestan. Se exploró además la memoria tecnológica del centro y se efectuó una revisión bibliográfica en diferentes fuentes de información para la preparación del estudio, tales como: Internet, publicaciones periódicas y no periódicas, libros, profesionales de la información, Intranet del CIGET y otros, con el fin de conocer las características de la gestión de la información y de la gestión del conocimiento. 
Se utilizó en todo momento el método de la observación para indagar los aspectos concernientes al quehacer del CIGET Guantánamo, el método histórico-lógico con el propósito de plasmar los detalles de la gestión de la información y la gestión del conocimiento y el método de análisis-síntesis para analizar el conjunto como cada parte de los procesos.

\section{Análisis y discusión}

Si se analiza y parafrasea la definición brindada por Goodman (1985, p.95) se entiende que la Gl se encarga de obtener la información a un precio óptimo, en el lugar (fuente) adecuado, por la persona (especialista) correcta, en el momento idóneo (cuando el usuario/cliente lo necesita); aunque es deber del especialista de la información adelantarse a las necesidades de sus usuarios y crear productos novedosos para ellos.

En el CIGET Guantánamo se realiza gestión de la información en acciones tan habituales como el servicio de referencia, que se encarga de responder cualquier pregunta o de resolver un problema de un usuario y/o cliente, relacionada o no con ciencia, tecnología y medio ambiente y en acciones complejas que incluyen servicios de alto valor agregado.

Por su parte la GC permite obtener el conocimiento presente en las personas y el que se genera en la organización para su posterior generalización.

Se realiza GI para adquirir información y ponerla a disposición de un usuario o cliente por medio de un servicio y en la elaboración de cada producto, y se aplica la GC cada vez que se comparte el conocimiento adquirido, en forma de diferentes acciones, es decir en los mismos productos y servicios.

La gestión del conocimiento y la gestión de la información vistas como partes de un sistema en el CIGET Guantánamo, donde cada una depende de la otra, son procesos indispensables para una organización de cualquier tipo, porque proporcionan información necesaria para: la toma de decisiones, la obtención de beneficios y la preservación, generalización y enriquecimiento del conocimiento organizacional.

A continuación se presentan las diferentes expresiones donde se evdencian la Gl y la GC en el CIGET Guantánamo. Primeramente se enumeran los productos y servicios informacionales dirigidos a los especialistas del CIGET y luego los que se brindan a los usuarios y clientes, es decir al personal externo que los solicita.

\subsection{Productos y servicios informacionales de uso interno en el centro}

Conformados por:

- Préstamo de documentos, periódicos, revistas, libros, mapas, diccionarios etc., y servicios bibliotecarios tradicionales (préstamo interbibliotecario, servicio de sala de lectura, entre otros).

- Localización de documentos a través del correo, el teléfono etc. por parte del especialista en otra unidad de información.

- Orientación de cursos, talleres, conferencias, seminarios y capacitaciones de diverso tipo sobre diversas temáticas en las áreas del CIGET y otras entidades del territorio guantanamero.

- Creación y mantenimiento de la intranet corporativa del CIGET y del portal de la ciencia guantanamera.

- Edición y publicación de artículos científicos en la revista electrónica Hombre, Ciencia y Tecnología.

- Organización de eventos de Ciencia de la información, Propiedad industrial y Gestión tecnológica TecnoGest.

- Elaboración de compendios informativos sobre diversas temáticas, manuales de procedimientos de trabajo, de mejores prácticas y de trabajos de consultorías organizacionales. 
- Creación y mantenimiento de memoria tecnológica y de la biblioteca digital.

- Transferencia de tecnologías y Alianzas estratégicas (con otros centros similares al CIGET Guantánamo y con otros especialistas de la ciencia, la tecnología y el medio ambiente).

- Participación en actividades de capacitación externa (en otras provincias de Cuba y en otros países)

- Participación en eventos y congresos.

- Obtención de vídeos educativos de temáticas variadas.

- Creación de bases de datos de varias temáticas

- Asignación de tutores a adiestrados y personal en servicio social.

- Elaboración de productos de información para los usuarios internos (trabajadores) del CIGET, tales como: boletines, búsquedas simples, traducciones, compendios informativos, perfiles de país y otros.

- Creación y moderación de artículos para la enciclopedia colaborativa cubana (Ecured)

- Socialización de las mejores prácticas de trabajo, en reuniones técnicas de trabajo o en talleres entre especialistas de un mismo grupo de trabajo y entre especialistas de diferentes áreas.

- Trabajo diario en las áreas de contabilidad, recursos humanos, operaciones, comercial, además de la secretaría.

\subsection{Productos y servicios informacionales para otras organizaciones o personales naturales}

Conformados por:

- Préstamo de documentos, periódicos, revistas, libros, mapas, diccionarios etc. en sala de lectura del CIGET y procesos tradicionales de bibliotecas.

- Localización de documentos a través del correo, el teléfono etc. por parte del especialista en otra unidad de información.

- Desarrollo de cursos, talleres, conferencias, seminarios y capacitaciones de diverso tipo sobre temáticas afines a la ciencia, la tecnología y el medio ambiente en las áreas del CIGET y otras entidades del territorio guantanamero.

- Creación y mantenimiento del portal de la ciencia guantanamera.

- Edición y publicación de artículos científicos en la revista electrónica Hombre, Ciencia y Tecnología.

- Obtención de vídeos educativos de temáticas variadas

- Creación de multimedias, páginas Web, intranet y otros.

- Elaboración de manuales de procedimientos de trabajo, y manuales de mejores prácticas.

- Servicio de consultorías organizacionales (sistema documental, sistema de archivo, sistemas de información, sistemas de comunicación, gestión de la innovación, gestión ambiental, gestión de la calidad, gestión del capital humano, seguridad y salud en el trabajo, etc.) 
- Organización y desarrollo de eventos científicos, para el intercambio de experiencias y conocimientos.

- Búsquedas de información a proyectos territoriales.

- Elaboración de bibliografías, listas de contenido, fototeca, videoteca, elaboración de diagramas de flujo, matrices DAFO y creación de bases de datos de varias temáticas.

- Asesoramiento en la creación de artículos científicos para publicar en revistas científicas.

- Elaboración de productos de información, vigilancia tecnológica, inteligencia empresarial entre otros tales como: boletines, búsquedas simples, traducciones, compendios informativos, perfiles estratégicos, estudios de tendencias, estudios de mercado y otros.

\subsection{Análisis de los produtos y servicios informacionales centrales generados}

Los efectos de la aplicación de los enfoques de la Gl y de la GC se traducen y se evidencian en cuatro productos y servicios informacionales centrales generados por el CIGET:

- Compendios informativos.

- Boletines informativos.

- Eventos.

- Cursos.

La elaboración y generación de los mismos se desarrolla bajo procedimientos y acciones pre-establecidas, que se detallan a continuación.

\subsubsection{Elaboración de compendios y boletines informativos}

En ambos casos se realiza un análisis de la temática, se establece la estrategia de búsqueda, se seleccionan las fuentes, se procede a la búsqueda de información, se realiza el tratamiento de la información obtenida (traducción, organización de bibliografía, lista de contenido), se organiza la información y se pone a disposición del usuario/cliente, puede entregarse en diversos formatos.

Los compendios comprenden temáticas relacionadas con la ciencia de montaña y sobre temas del café principalmente.

Las temáticas de los boletines han estado encaminadas a la gestión de riesgos de desastres y a temas de interés para los directivos de las diferentes empresas de la ciudad de Guantánamo.

El contenido temático, de los compendios realizados para un centro especializado en la ciencia de montaña, abordaba los siguientes tópicos:

- Orquídeas.

- Frutales arbóreos sub explotados.

- Las péctinas como gelificantes y estabilizantes.

- Aplicación de sistemas agroforestales en los cultivos de cacao y café.

- Zonificación agroecológica de los cultivos.

- Tecnologías de cultivo de arroz en secano. 
- Tecnologías de producción de frijol.

- Tecnologías para la producción de posturas de cocotero.

- Tecnologías para la recuperación de áreas degradadas.

- Vegetación en bosques fragmentados.

- Tecnologías para el aprovechamiento de residuos sólidos y líquidos.

- Manejo de desechos peligrosos.

- Plaguicidas y bioplaguicidas.

- Toxina de alacrán.

- Medidas de estimación de biodiversidad.

\subsubsection{Gestión de la información para boletines informativos}

Los boletines le permiten al usuario/cliente recibir información de manera sistemática, actualizada y confiable sobre uno o varios temas de su interés que le sirven para adelantarse a la competencia, identificar nichos de mercado, conocer a los líderes en un área determinada, identificar las amenazas y eliminarlas, incrementar las fortalezas, aprovechar las oportunidades, etc. Todo con el uso de la información que se recopila, procesa y se pone a disposición del usuario/cliente.

Se elaboran con secciones fijas, tales como efemérides, curiosidades, noticias, hechos de interés, análisis económicos, etc. Posibilitan la toma de decisiones operativas y el desarrollo de actividades de l+D+i, con ellos se socializa información de vital importancia y se garantiza el análisis y gestión eficiente de los recursos con que se cuenta.

\subsubsection{Gestión de eventos y cursos}

Los eventos del CIGET están referidos principalmente a la Propiedad Intelectual, durante el mes de abril (en saludo al día de la Propiedad Intelectual que se celebra cada 26 de abril); a la Ciencia de la Información en el mes de junio (en saludo al día del bibliotecario que se celebra el día 7 de ése mes); y a la Tecnología, cuando octubre se celebra TecnoGest, evento magno del centro.

Estos eventos se preparan con varios meses de antelación, por su comité organizador, para ello se analizan las temáticas principales que se abordarán y se preparan conferencias magistrales, se invitan expertos en diferentes temas, se elabora el programa científico, se desarrollan trabajos por comisiones, donde predomina el intercambio de ideas, en algunas ediciones el evento tienen carácter competitivo, para ello se platean en la convocatoria, diversas temáticas para desarrollar trabajos de investigación.

Al finalizar el evento se recogen encuestas que se entregan previamente como medio de retroalimentación, para conocer las sugerencias de los participantes, sus opiniones, etc.

Los cursos son impartidos tanto por especialistas del CIGET o por especialistas invitados de otras entidades que se consideran expertos en un área determinada. Las clases que se imparten se preparan en no menos de 96 horas, se efectúa una exhaustiva búsqueda de información sobre los temas a impartir, y se siguen los pasos del proceso docente-educativo. Además se desarrollan clases prácticas, actividades de participación, evaluaciones diarias y ejercicios de evaluación final. También se entregan diplomas de participación, para lo cual se controla la asistencia. Estas clases pueden ser impartidas por uno o más profesores.

\section{Conclusiones}

En el trabajo desarrollado en el Centro de Información y Gestión Tecnológica (CIGET) de Guantánamo se pueden identificar varias expresiones de la gestión de la información y gestión del conocimiento para los diversos usuarios y clientes del centro. 
Los efectos de la aplicación de los enfoques de la Gl y de la GC se traducen y se evidencian en cuatro productos y servicios informacionales centrales: compendios informativos, boletines informativos, eventos, y cursos.

Finalmente consideramos que para la sostenibilidad y evolución de estos productos y servicios, es necesario considerar las siguientes acciones estratégicas:

- Incrementar las acciones de gestión de la información y gestión del conocimiento en el CIGET Guantánamo.

- Utilizar nuevas herramientas que apoyen la gestión de la información y la gestión del conocimiento y crear algunas.

- Incentivar la elaboración de trabajos relacionados con la gestión de la información y la gestión del conocimiento.

- Divulgar los temas de gestión de la información y gestión del conocimiento para facilitar su comprensión y la optimización de su aplicación.

- Incrementar los espacios de socialización de conocimientos.

\section{Bibliografía}

Aja, L. (2002) Gestión de información, gestión del conocimiento y gestión de la calidad en las organizaciones [Versión electrónica]. ACIMED, vol.10, no 5. Disponible en: http://scielo.sld.cu/scielo.php?pid=S102494352002000500004\&script=sci arttext

Artiles, S. (2009) La gestión documental, de información y el conocimiento en la empresa. El caso de Cuba. [Versión electrónica]. ACIMED, vol.19, oㅡ 5 Recuperado de: http://scielo.sld.cu/scielo.php?script=sci arttext\&pid=S102494352009000500002

Colectivo de autores. (2009). Inteligencia empresarial. Qué y cómo. Consultoría Biomundi/IDICT. La Habana

Correa, G., Rosero, S., Segura, H. (2008) Diseño de un modelo de gestión del conocimiento para la Escuela Interamericana de Bibliotecología. [Versión electrónica]. Rev. Interam. Bibliot., vol. 31, № 1, p. 85-108, Recuperado de: http://eprints.rclis.org/12325/1/ARTICULO4.pdf

Dans, E. (2006) El departamento de sistema de información y su función corporativa: retos de futuro en investigación. [Versión electrónica]. Recuperado de: http://profesores.ie.edu/enrique dans/download/retosdefuturo.pdf

González, A., Parés, M. (2012) Gestión del Conocimiento en Cuba: diseminación de sus resultados de investigación, de 1997 2010. [Versión electrónica]. Ciencias de la Información. Vol. 43, № 3. 23- 32. Recuperado de:

http://cinfo.idict.cu/cinfo/article/view/436/pdf

León, M., Castañeda, D., Sánchez, I. (2007) La gestión del conocimiento en las organizaciones de información: procesos y métodos para medir. [Versión electrónica]. Acimed, vol. 15, № 3. Recuperado de:

http://bvs.sld.cu/revistas/aci/vol15 3 07/aci02307.htm

León, M., Ponjuán, G., Rodríguez, M. (2006) Procesos estratégicos de la gestión del conocimiento. [Versión electrónica]. Acimed, vol. 14 2. Recuperado de: http://bvs.sld.cu/revistas/aci/vol14 2 06/aci08206.htm

Leyva, J. (2013) Modelo para diagnosticar los factores clave de la implantación de sistemas de gestión del conocimiento para el desarrollo local. [Versión electrónica]. Economía de Cuba, no 178. Recuperado de: http://www.eumed.net/cursecon/ecolat/cu/2013/sistemas-de-gestion-del-conocimiento-para-el-desarrollo-local.html

Monagas, M. (2012) El capital intelectual y la gestión del conocimiento. [Versión electrónica]. Ing. Ind., vol. 33, № 2. Recuperado de: http://scielo.sld.cu/scielo.php?pid=S1815-59362012000200006\&script=sci arttext

Muñoz, A., Pulgarín, A. (2009) La gestión de la información en las empresas de Extremadura. [Versión electrónica]. Revista Española de Documentación Científica, vol. 32, no 4, 107-132. Recuperado de: http://redc.revistas.csic.es/index.php/redc/article/download/517/575

Núñez, I. (2004) La gestión de la información, el conocimiento, la inteligencia y el aprendizaje organizacional desde una perspectiva socio-psicológica. [Versión electrónica]. Acimed, vol. 12, № 3. Recuperado de: http://bvs.sld.cu/revistas/aci/vol12 3 04/aci04304.htm

Palacios, D., Garrigós, F. (2006) Propuesta de una escala de medida de la gestión del conocimiento en las industrias de biotecnología y telecomunicaciones. [Versión electrónica]. Investigaciones europeas de dirección y economía de la empresa, vol. 12, no 1, 207-224. Recuperado de: http://dialnet.unirioja.es/descarga/articulo/2010926.pdf 
Pereira, H. (2011) Implementación de la gestión del conocimiento en la empresa. [Versión electrónica].ำ 135. Recuperado de: http://www.cegesti.org/exitoempresarial/publicaciones/publicacion 135310111 es.pdf

Pérez, A. (2008) Conceptos y posibilidades de la gestión del conocimiento en la Universidad. [Versión electrónica]. Gestión Universitaria, vol. 1, no 1,. Recuperado de: http://www.gestuniv.com.ar/gu 01/v1n1a2.htm

Pérez, J. (2003) La Era del Conocimiento. El Mar y la Montaña. Guantánamo.

Pérez, M. (2003) Gestión del conocimiento y documentación digital: un estudio de caso. [Versión electrónica]. Hipertext, ํㅡㄴ 1 . Recuperado de: http://www.upf.edu/hipertextnet/numero-1/gestion conoc.html

Soto, M., Barrios, N. (2006) Gestión del conocimiento. Parte I. Revisión crítica del estado del arte. [Versión electrónica]. Acimed, vol. 14, no 2. Recuperado de: http://bvs.sld.cu/revistas/aci/vol14 2 06/aci04206.htm

\section{Datos de las autoras}

\section{Zunilka Limonta Favier}

Licenciada en Bibliotecología y Ciencia de la Información, graduada en la Universidad de Camagüey, promoción 2008, profesor Instructor. Diplomada en Comunicación organizacional. Especialista en análisis, procesamiento y servicios de información en Centro de Información y Gestión Tecnológica. Especialista en Inteligencia empresarial, Cuba.

zunilka@ciget.gtmo.inf.cu

\section{Evelyn Milagro Otero Caballero}

Licenciada en Bibliotecología y Ciencia de la Información, graduada en el Instituto Superior Minero Metalúrgico de Moa, Holguín, promoción 2010. Especialista en análisis, procesamiento y servicios de información en Centro de Información y Gestión Tecnológica, Guantánamo, Cuba.

evelyn@ciget.gtmo.inf.cu

\section{Elizabeth Álvarez Cambas}

Licenciada en Psicología, graduada en la Universidad de Oriente, Santiago de Cuba. Directora y consultora organizacional de Centro de Información y Gestión Tecnológica, Guantánamo, Cuba. elizabeth@ciget.gtmo.inf.cu

\section{Yaima Ripoll Moreno}

Licenciada en Bibliotecología y Ciencia de la Información, graduada en el Instituto Superior Minero Metalúrgico de Moa, Holguín, promoción 2010, Especialista en análisis, procesamiento y servicios de información en Centro de Información y Gestión Tecnológica, Bayamo, Granma.

yaima@ciget.granma.inf.cu

Recibido - Received : 2014-10-30

Aceptado - Accepted: 2015-03-30

\section{$(\mathrm{cc})$ EY}

This work is licensed under a Creative Commons Attribution 4.0

United States License.

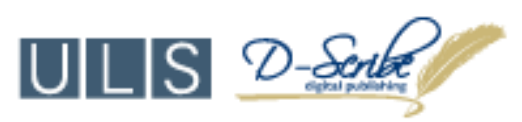

This journal is published by the University Library System of the University of Pittsburgh as part of its D-Scribe Digital Publishing Program and is cosponsored by the University of Pittsburgh Press. 